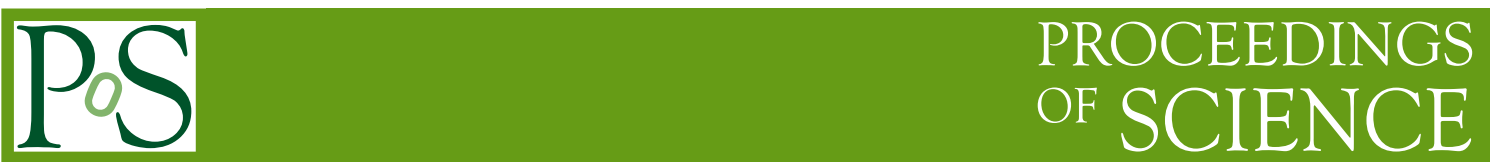

\title{
Global fits of the MSSM with GAMBIT
}

\author{
Anders Kvellestad*, on behalf of the GAMBIT collaboration \\ Department of Physics, Imperial College London, Blackett Laboratory, Prince Consort Road, \\ London SW7 2AZ, UK \\ Department of Physics, University of Oslo, N-0316 Oslo, Norway \\ E-mail: a.kvellestadeimperial.ac.uk
}

\begin{abstract}
The minimal supersymmetric standard model is a popular and well-motivated extension of the standard model. As such, it has been constrained by a large number of different experimental searches. To truly assess the impacts of these experiments on the model one must perform a global fit, scanning over the multi-dimensional parameter space and combining all the data in a statistically rigorous manner. In this talk, I presented results from global fits of supersymmetric models performed with GAMBIT, the Global and Modular Beyond-the-Standard-Model (BSM) Inference Tool. I showed MSSM results from the latest GAMBIT papers, as well as exciting preliminary results from a dedicated study of the collider constraints on the electroweakino sector.
\end{abstract}

The 39th International Conference on High Energy Physics (ICHEP2018)

4-11 July, 2018

Seoul, Korea

${ }^{*}$ Speaker. 
Initial note: In this conference paper I present preliminary results from an analysis of collider constraints on the MSSM electroweakino sector. This work has recently been finalized and is presented in full in Ref. [1].

Weak scale supersymmetric extensions of the standard model can solve the large hierarchy problem, explain dark matter (DM), and achieve gauge coupling unification. In recent years, the minimal supersymmetric standard model (MSSM) has come under heavy pressure from a vast array of experiments in particle physics and astrophysics. To correctly assess their impact on the model, one must perform a statistically rigorous combination of the data in a global fit.

The Global and Modular BSM Inference Tool (GAMBIT) is both a state-of-the-art tool for performing global fits in essentially any BSM theory, and a collaboration using it to perform global fits in a wide variety of BSM theories. The GAMBIT code $^{1}$ is public, and is accompanied by six component manuals [2, 3, 4, 5, 6, 7]. Physics studies so far cover singlet DM models [8, 9, 10], axion models [11], and various parameterisations of the MSSM [12, 13]. Here, I present results from the published MSSM global fits, along with intriguing preliminary results from a dedicated fit of the MSSM electroweakino sector to recent LHC results.

Fit results for the constrained MSSM (CMSSM) are shown in the top row of Fig. 1. One can see three active mechanisms to avoid DM overabundance: ${ }^{2}$ stop co-annihilation, chargino coannihilation and resonant annihilation through a heavy Higgs funnel. Note that stau co-annihilation, which has previously appeared in CMSSM global fits, is now ruled out at the 95\% confidence level (CL). The overall best fit point is a stop co-annihilation scenario with stop and neutralino masses of around $600 \mathrm{GeV}$. The rightmost panel shows that the chargino co-annihilation and Higgs-funnel regions can be fully probed by future direct detection experiments. The bottom row of Fig. 1 shows fit results for a weak-scale MSSM parameterisation with seven free parameters (MSSM7). We find lightest neutralinos that are Higgsino dominated, bino dominated and a mixture of the two. ${ }^{3}$ The best-fit point is a chargino co-annihilation scenario with chargino and neutralino masses around $260 \mathrm{GeV}$ and a mass difference of around $10 \mathrm{GeV}$. The entire chargino co-annihilation and light Higgs funnel preferred regions will be probed by future direct detection experiments.

In Fig. 2 we show preliminary results from a global fit focused on the electroweakino sector of the MSSM, where we decouple all SUSY states other than the neutralinos and charginos. We focus our investigation on collider searches, with the aim of identifying what impact these searches, which are typically interpreted in simplified models, have had on the general MSSM electroweakino sector. Here we include the rigorous ColliderBit implementation of almost all relevant LHC searches for promptly decaying electroweakinos, using $36 \mathrm{fb}^{-1}$ of $13 \mathrm{TeV}$ data. For the CMS analyses that provide approximate background covariance matrices, we follow the "simplified likelihood" approach introduced in [16] to make use of this information. The two ATLAS searches in [15] and [14] both target final states with two or three leptons. However, the overlap between the final datasets for the two analyses is relatively small [14]. In Fig. 2 we show the fit results obtained when we only include the recursive jigsaw analysis from [14] (left panel) in our total likelihood function, and when both analyses are included as independent likelihood contributions

\footnotetext{
${ }^{1}$ https://gambit.hepforge.org

${ }^{2}$ We apply the relic density constraint as an upper bound only, as it is possible that a fraction of DM may exist as another state (e.g. axions).

${ }^{3}$ Winos cannot dominate the lightest neutralino, since $M_{2} \approx 2 M_{1}$ in the MSSM7.
} 

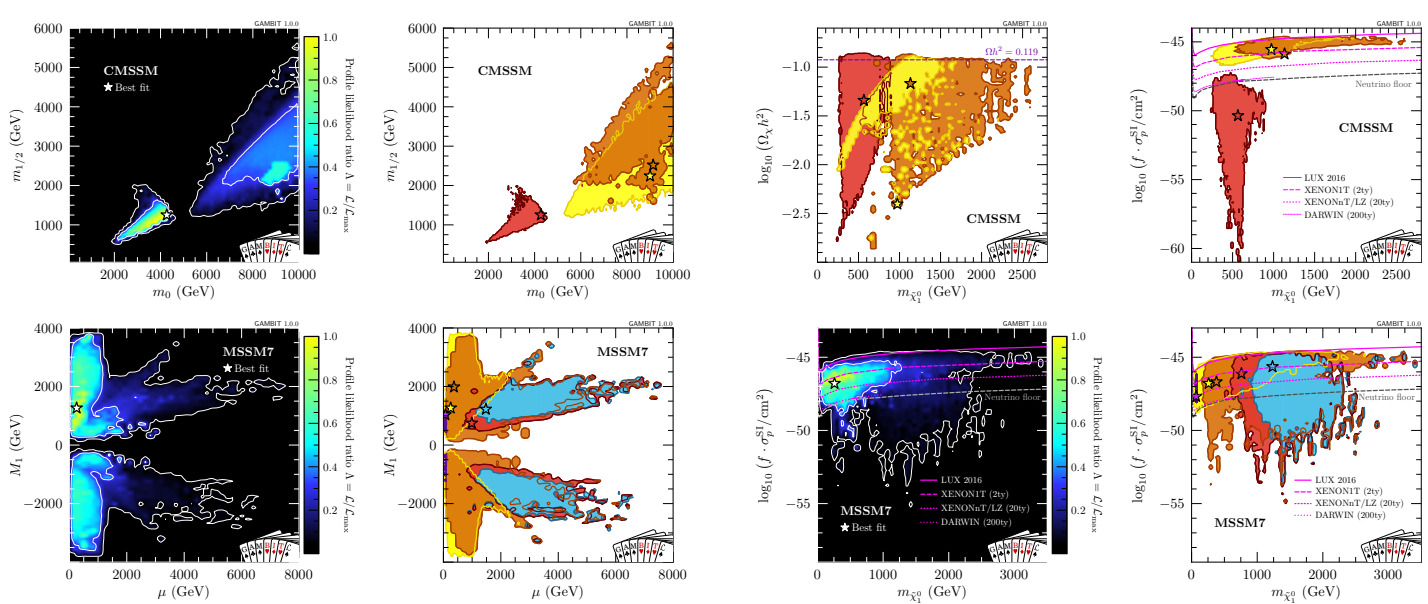

$\tilde{t}_{1}$ co-annihilation

$A / H$ funnel $\square \tilde{\chi}_{1}^{ \pm}$co-annihilation

$\tilde{b}_{1}$ co-annihilation

$h / Z$ funnel

Figure 1: Results from the CMSSM (top row) and MSSM7 (bottom row) global fits, taken from Refs. [12, 13]. The dark-background panels depict the profile likelihood ratio, with white contour lines indicating the $68 \%$ and $95 \%$ confidence level (CL) regions. The white-background panels show the mechanisms for depleting the relic density of dark matter to or below the measured value, within the $95 \% \mathrm{CL}$ profile likelihood regions. The spin-independent nuclear scattering cross-sections are rescaled according to the predicted fraction of the relic density in neutralinos $(f)$. See the text for further details.
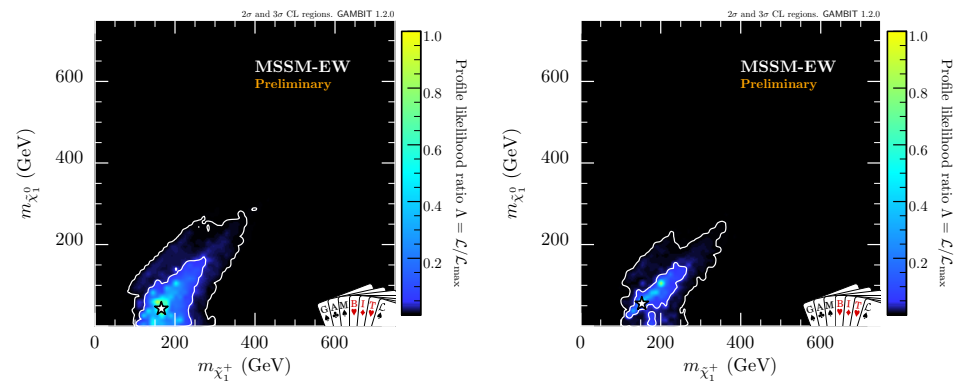

Figure 2: Preliminary results from the electroweakino fit. In the left panel only one ([14]) of the two ATLAS searches in two- and three-lepton final states $([15,14])$ is included in the total likelihood function, while in the right panel both searches have been included. White contours correspond to $95 \%$ and $99.7 \%$ CL regions.

(right panel). Our fit also takes into account constraints on chargino and neutralino production at LEP, and limits on the invisible decay widths of the $Z$ and the $125 \mathrm{GeV}$ Higgs.

The preliminary result in Fig. 2 is quite intriguing, as we see closed $3 \sigma$ CL contours, suggesting the presence of a signal. The result should be interpreted with great care until the overall goodness of fit of the highlighted models has been studied in detail.

\section{Acknowledgments}

I gratefully acknowledge the rest of the GAMBIT Collaboration, as well as Matthias Danninger, Rose Kudzman-Blais, Andreas Petridis, Abhishek Sharma and Yang Zhang for contributions to the work presented here. I also acknowledge PRACE for awarding GAMBIT access to 
Marconi at CINECA, Italy.

\section{References}

[1] GAMBIT: P. Athron et. al., Combined collider constraints on neutralinos and charginos, arXiv:1809.02097.

[2] GAMBIT Collaboration: P. Athron, C. Balázs, et. al., GAMBIT: The Global and Modular Beyond-the-Standard-Model Inference Tool, Eur. Phys. J. C 77 (2017) 784, [arXiv: 1705.07908 ].

[3] GAMBIT Scanner Workgroup: G. D. Martinez, J. McKay, et. al., Comparison of statistical sampling methods with ScannerBit, the GAMBIT scanning module, Eur. Phys. J. C 77 (2017) 761, [arXiv:1705.07959].

[4] GAMBIT Collider Workgroup: C. Balázs, A. Buckley, et. al., ColliderBit: a GAMBIT module for the calculation of high-energy collider observables and likelihoods, Eur. Phys. J. C 77 (2017) 795, [arXiv:1705.07919].

[5] GAMBIT Flavour Workgroup: F. U. Bernlochner, M. Chrząszcz, et. al., FlavBit: A GAMBIT module for computing flavour observables and likelihoods, Eur. Phys. J. C 77 (2017) 786, [arXiv:1705.07933].

[6] GAMBIT Dark Matter Workgroup: T. Bringmann, J. Conrad, et. al., DarkBit: A GAMBIT module for computing dark matter observables and likelihoods, Eur. Phys. J. C 77 (2017) 831, [arXiv:1705.07920].

[7] GAMBIT Models Workgroup: P. Athron, C. Balázs, et. al., SpecBit, DecayBit and PrecisionBit: GAMBIT modules for computing mass spectra, particle decay rates and precision observables, Eur. Phys. J. C 78 (2018) 22, [arXiv: 1705. 07936].

[8] GAMBIT Collaboration: P. Athron, C. Balázs, et. al., Status of the scalar singlet dark matter model, Eur. Phys. J. C 77 (2017) 568, [arXiv:1705.07931].

[9] P. Athron, J. M. Cornell, et. al., Impact of vacuum stability, perturbativity and XENONIT on global fits of $\mathbb{Z}_{2}$ and $\mathbb{Z}_{3}$ scalar singlet dark matter, Eur. Phys. J. C78 (2018) 830, [arXiv:1806.11281].

[10] GAMBIT: P. Athron et. al., Global analyses of Higgs portal singlet dark matter models using GAMBIT, arXiv:1808.10465.

[11] S. Hoof, F. Kahlhoefer, P. Scott, C. Weniger, and M. White, Axion global fits with Peccei-Quinn symmetry breaking before inflation using GAMBIT, arXiv:1810.07192.

[12] GAMBIT Collaboration: P. Athron, C. Balázs, et. al., Global fits of GUT-scale SUSY models with GAMBIT, Eur. Phys. J. C 77 (2017) 824, [arXiv:1705.07935].

[13] GAMBIT Collaboration: P. Athron, C. Balázs, et. al., A global fit of the MSSM with GAMBIT, Eur. Phys. J. C 77 (2017) 879, [arXiv:1705.07917].

[14] ATLAS Collaboration: M. Aaboud et. al., Search for chargino-neutralino production using recursive jigsaw reconstruction in final states with two or three charged leptons in proton-proton collisions at $\sqrt{s}=13 \mathrm{TeV}$ with the ATLAS detector, arXiv:1806.02293.

[15] ATLAS Collaboration: M. Aaboud et. al., Search for electroweak production of supersymmetric particles in final states with two or three leptons at $\sqrt{s}=13 \mathrm{TeV}$ with the ATLAS detector, arXiv: 1803.02762.

[16] CMS Collaboration, Simplified likelihood for the re-interpretation of public CMS results, CMS-NOTE-2017-001, 2017. 